\title{
51
}

\section{The application of Integrated Intelligent Systems (IIS) to production scheduling}

\author{
Kesheng Wang and Wenfen Lin \\ Department of Production and Quality Engineering \\ NTNU, \\ N-7034 Trondheim, Norway \\ Tel. +4773597119 \\ Fax. +4773597117 \\ e-mail: kesheng.wang eprotek.ntnu.no
}

\begin{abstract}
One of the most important issues in the process of development and implemention of a manufacturing system is production scheduling. Different scheduling rules and algorithms have been widely developed and used for supporting decision-making in production scheduling. However, in practice, it is very difficult for a user to understand, build or select a suitable and efficient model for the system considered. Hence, an intelligent system is to be built for problem solving. This paper describes how the concept of Integrated Intelligent Systems (IIS), which is developed by Wang (1995) at NTNU in order to meet requirements for higher efficiency, great flexibility, better product quality, and low costs in manufacturing, should be applied to integrate different software packages to solve production scheduling problems. The prototype system developed may be used for daily production scheduling in a manufacturing environment.
\end{abstract}

\section{Keywords}

Intelligent Manufacturing Systems, Integrated Intelligent Systems, distributed AI, Expert System (ES), production scheduling. 
As a well known fact, the modern manufacturing industry does not only need high technology, but also the expertise in various different manufacturing domains in order to meet requirements for higher efficiency, great flexibility, better product quality, and lower costs. The core of this manufacturing technology is integration of domains of specific knowledge, that is, the integration of industry design, manufacturing, planning, managing and marketing activities as well as decisionmaking automation. The development and application of Integrated Intelligent Systems (IIS) will speed up the manufacturing process and improve the production efficiency, product quality, consequently, and it will enhance company's competition ability in the international market. For this purpose, Integrated Intelligent Systems (IIS) has been developed at Department of Production and Quality Engineering by Prof. Wang. [17] The concept and structure is well suitable for small and medium size companies to realize "balanced automation" in their manufacturing environment.

Production scheduling remains as an important aspect in manufacturing systems. In the international market, it is a very important that companies have to focus on delivering their products to customers within shortest time and higher quality. It becomes very critical to use company's resource effectively and flexibly. In production scheduling, different conditions, such as the importance and the availability of jobs, and different objectives, such as minimizing average lateness, makespan, average flowtime, etc., have to be considered. In the past, those working in the field of operations research have made an important contribution. (Barker 1974, French 1982, Gere 1966). Scheduling is seen primarily as combinatory optimization problem: the question of which is most effective sequence for orders as to due-date performance and utilization is addressed. In attempting to solve this problem, simplifications have to be made as regards to concrete constraints. This leads to low acceptance of the proposed solutions in actual practice in industry. In contrast, the contribution of production researchers is characterized by the endeavor to develop heuristics and dispatching rules under practical constraints leading to acceptable approximations in a reasonable computing time. (Alexander 1978, Balckstone 1982, Conway 1967, Kusiak 1992, , Panwallkar 1977, Pinedo 1995). Despite the above-mentioned attempts to simplify the production process, the production scheduling problem is still an important research in manufacturing environment.

Different scheduling models and algorithms have been widely developed and used to support decision-making in many industries including manufacturing, petroleum, steel, forestry, communications and banking. However, difficulties have been encountered with formulation of models, management of data, and interpretation of the results. Because the complexity of models, the use of these models has been limited to professionals who have enough domain knowledge in mathematics, optimization, and Operation Research (OR). In practice, a user may 
not know how to build or select a model required for the system considered. For example a manager who determines a production schedule may not know which kind of models is appropriate for solving the problem, or may not be aware of the best solution approaches. Hence, an intelligent system is needed to be built for problem solving.

Integrated Intelligent Systems provide new sight to production scheduling. The increasing complexity of selecting the right procedure, understanding the procedure, integrating the scheduling system with other applications and solving an instant, can be dramatically reduced by the use of the IIS conception. This paper describes how the concept of Integrated Intelligent Systems should be applied to integrate different software packages to solve production scheduling problems. The prototype system developed may be used to daily production scheduling in a manufacturing environment.

In this paper, Section 2 gives a brief survey of IIS and some advantages of using it in manufacturing environments. Section 3 illustrates the approach of solving production scheduling problems which is the theoretical base for developing the scheduling expert system. Section 4 focuses on the development of the subsystems of IIS. A case study is also demonstrated in section 5 and a conclusion is put in section 6.

\section{INTEGRATED INTELLIGENT SYSTEMS (IIS) APPROACH}

Integrated Intelligence Systems (IIS) have significant advantages over conventional structured computer programs in applications such as CIM. The structure of IIS is shown in Figure 1. IIS is a knowledge integration environment, which consists of several numerical computation packages, symbolic reasoning systems, database management systems, computer graphics packages, multimedia techniques, neural networks, domain expert systems, and a meta-system. The integrated software environment allows the running of programs written in different languages and communication among programs as well as the exchange of data between programs and database. These separated subsystems need not be intelligent, but the supervising system, the meta-system, should be intelligent. The meta-system manages the selection, co-ordination, operation and communication of these programs. The conception of IIS is perfectly suitable for manufacturing integration. The existing computer systems, such as CAD, CAE, CAPP, CAM, etc. in a manufacturing enterprise might be integrated together through an intelligent meta-system. One major advantage is that Integrated Intelligent Systems offer a modular architecture for building large programs. Knowledge in the form production rules may be added, deleted or modified in the knowledge base, without any change in the control structure. An Integrated Intelligent System's ability to perform symbol manipulation permits users to choose a variety of representation schemes for components. 


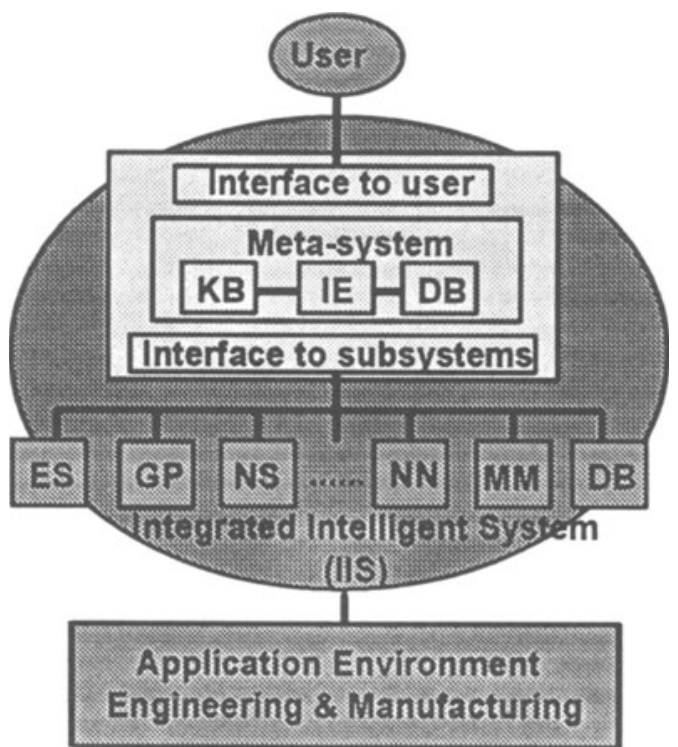

KB: Knowledge Base, IE: Inference Engine, DB: Data Base, ES: Expert Systems, GP: Graphics Packages, NS: Numerical Software, NN: Neural Networks, MM: Multi-Media systems.

Figure 1 The structure of Integrated Intelligent System (IIS)

The meta-system was developed by the use of KnowledgePro - an intelligent system development tool. The details on the meta-system are presented in the following references (KnowledgePro, 1991).

PRODUCTION SCHEDULING

\subsection{Classification of production scheduling problem}

The pure sequencing problem is a specialized scheduling problem in which an ordering of the jobs completely determines a schedule. Moreover, the simplest pure sequencing problem is one in which there is a single resource or machine. The objective is to schedule $n$ jobs on one machine such that one or more performance measures are satisfied. Some of the widely used performance measures are minimizing the number of tardy jobs, earliness, lateness, tardiness and some cost functions. The solution approaches very greatly depending on where the problem is static or dynamic; deterministic or probabilistic; A scheduling problem is defined static if the arrival times of all the jobs are known 
in advanced. otherwise, it is classified as dynamic scheduling problem. On the other hand, a scheduling problem is called deterministic if all of the parameter such as processing times, setup times, etc. are known with certainty. Otherwise it is classified as probabilistic. (See Figure 2.) The decision tree for classification of production scheduling problem is considered as following: (1) Single Machine Scheduling. Each job consists a single operation. This is the case of simplest of all possible machine environments and is a special case of all other more complicated machine environment. (2) Parallel Machines Scheduling. More than one machine to perform the processing. and (3) Flow Shop Scheduling. There is a nature ordering of the machines in a shop. All parts flow in one direction. (see Figure 2.)

\subsection{Assumptions On Scheduling Problems}

We know that the real world is not so neat as we have envisaged, so it is necessary to make some assumptions to make the model more feasible. Follows are the six general assumptions for the whole system and assumptions for each shop type, respectively.

3.2.1 Assumptions on General Case: (1) Static Scheduling Problem. (2) Deterministic Scheduling Problem. (3) Independent Jobs. (4) SequenceIndependent Setup Times. (5) No Cancellation Allowed. (6) No Breakdown Allowed (the machines have no other tasks and it will be continually available until all jobs are completed).

\subsubsection{Assumptions on Individual Case:}

- Single Machine Scheduling: (1) Single-Operation Jobs. (2) One Machine Always Available. (3) No Preemption Allowed.

- Parallel Machine Scheduling (1) Single-Operation Jobs. (2) m Identical Machines Continuously Available. (3) Preemption Allowed.

- Flow Shop Scheduling: (1) Multiple-Operation Jobs. (2) m Different Machines Continuously Available. (3) No Preemption Allowed.

Here, preemption means the processing is not interrupted and a job is not removed from a machine before an operation is completed, i.e., no job has any priority.

\subsubsection{Objectives Handled}

After the assumptions above, the system is ready to be modeled. But before we establish it, we must know which kind of objective is our interest since this is nature in the real workshop for a manager to know his/her objectives. Follows are some objectives that are common used in real case. Baker 1974, Gere 1966, Gursel 1995, Bellman 1982).

1. Average Completion Time(C). 2. Average Lateness(L). The lateness of job is defined as the difference between job's completion time $C_{i}$ and 
job's due date $d_{i}$, i.e., $L_{i}=c_{i}-d_{i}$, which is positive when job is completed late and negative when it is completed early. 3. Average Waiting Time(W). 4. Average Flow Time(F). The sum of the completion time of $\mathbf{n}$ jobs is called flow time. It gives an indication of the total holding, or inventory, costs incurred by the schedule. 5. Average Tardiness(T). 6.Maximum Lateness(ML). 7. Maximum Tardiness(MT). 8. Number of Tardy Jobs(nT). 9. Minimum Makespan(Ms). The makespan is defined as max $\left(C_{1}, \ldots . C_{n}\right)$, is equivalent to the completion time of the last job to leave the system. 10. Minimize Maximum Cost of Processing(MM).

\subsubsection{Conditions Considered}

To make a decision in scheduling, we should also consider some shop conditions, these conditions are as follows,

1. Shop Configuration (Static and deterministic): a). Single Machine; b). Parallel Machines; c). Flow Shop.

2. Importance of Jobs: a). All jobs equally important; b). At least one job has different importance.

3. Availability of Jobs: a). All jobs available at time zero; b). At least one job is not available at time zero.

4. Preemption: a). Allowed; b). Not allowed.

5. Number of Machines/Processors: a). Single Machine; b).Multiple Machines

As described in section 2, IIS is configured with a meta-system as its core component and several subsystems connecting parallel to the meta-system. One of the advantages of such a kind of structure is to let system developers focus on the development of subsystems. The subsystems of production scheduling problem includes: (1) Scheduling expert system, (2) rules and algorithms packages.

\subsection{Scheduling expert system}

A rule-based knowledge representation scheme is selected to develop the prototype of production scheduling system. For convenience, the expert system is also written in KnowledgePro. When the consultation starts, the user is asked the following questions:

(1) Shop configuration; (2) Importance of jobs (equally importance or not);

(3) Objective of the user (Minimize makespan); (4) Availability of jobs (ready time $=0$, ready time $>0$ ). 
Depending on the responses specified by the user, the expert system selects the appropriate scheduling rule or algorithm to solve the problem. Having determined the procedure, an interface is provided between meta-system and user, thus enabling the user to solve instant of the problem by entering the necessary data in a user-friendly environment.

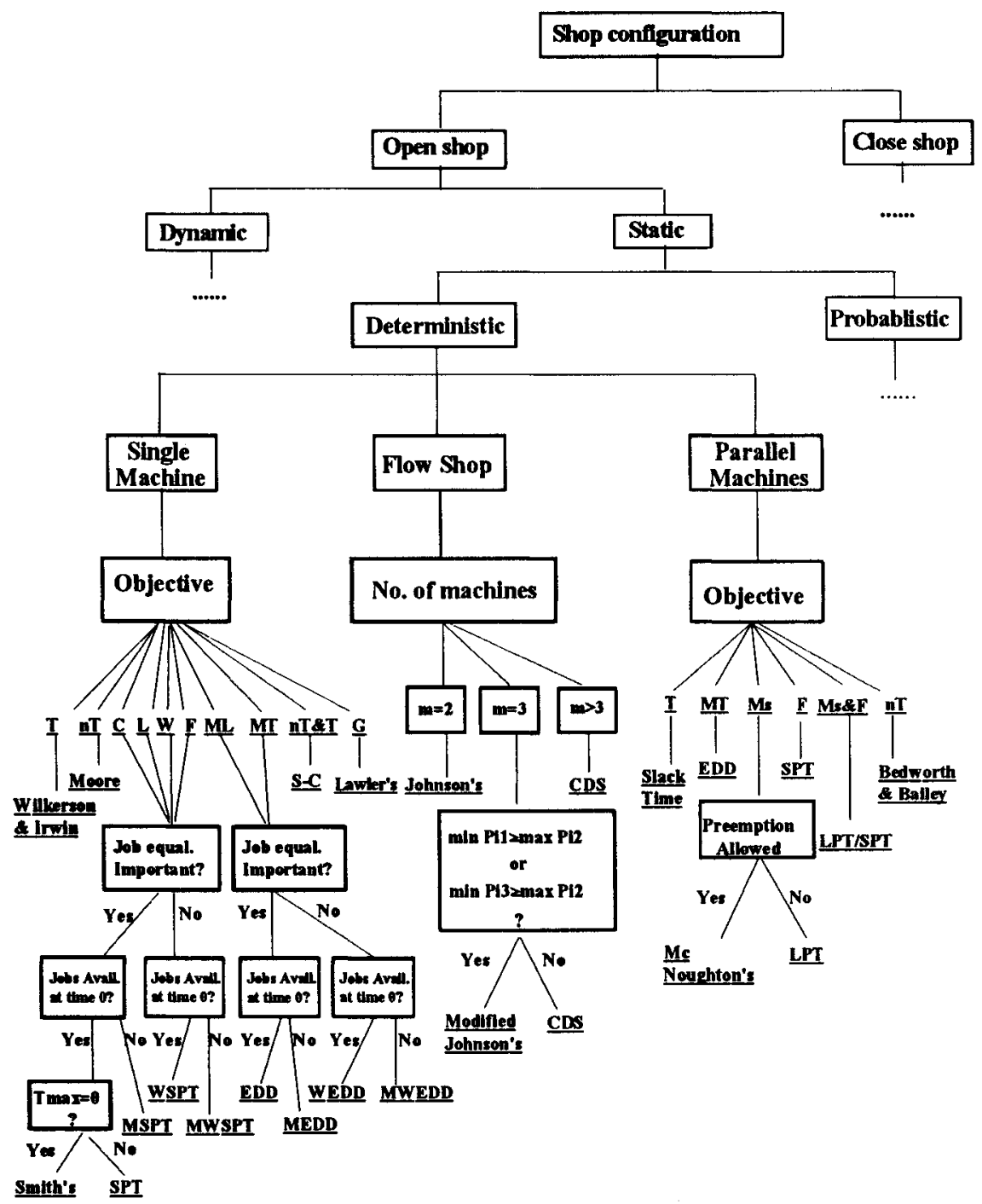

Figure 2 The decision tree of production scheduling problems 


\subsection{Rules and Algorithms packages}

The rules and the algorithms included in the production scheduling, and under what conditions they are chosen are shown in Figure 2 and listed as following:

(1) Shortest Processing Time (SPT); (2) Weighted SPT (WSPT); (3) Earliest Due Data (EDD); (4) Modified SPT (MSPT); (5) Modified WSPT (MWSPT); (6) Modified EDD (MEDD); (7) Modified WEDD (MWEDD); (8) Moore's Algorithm; (9) Smith's Algorithm; (10) Lawler's Algorithm; (11) Wilkerson-Irwing Algorithm; (12) S-C Algorithm; (13) Largest Processing Time (LPT)-Parallel Machines; (14) SPT - Parallel machines; (15) LPT-SPT - Parallel machines; (16) Bedworth and Bailey Algorithm; (17) Slack Time Rule; (18) EDD - parallel machines; (19) Mc Noughton's algorithm; (20) Johneson's algorithm (flow shop, 2 machines); (21) Modified Johson's algorithm; (22) C_D_S algorithm /Flow shop, m machines), ......., etc.

The rules and algorithms were developed in high-level language $\mathrm{C}$, spreadsheet Excel and Operation Research software Lindo, depending on the features and the complexity of nules and algorithms. The reason why we select these software may be:

(1) Ease of use; (2) Common-used in Companies; (3) PC-based platform.

Excel was used for the developing of simple rules (e.g. SPT) and for input data, C for complex rules (e.g., Johnson's algorithm) and Lindo for OP algorithms (e.g., integer programming). All these software are easily and rapidly integrated with the meta-system.

\section{SAMPLE CONSULTATION}

Johnson's Algorithm Applied in Two-Machine Flow Shop is used for demonstrate how the system to function. Suppose the user's shop is a two- machine flow shop with parameters shown in the following:

Table 1 Parameters of a two-machine flow shop

\begin{tabular}{c|cc}
\hline $\begin{array}{c}\text { Operation number } \\
i\end{array}$ & $\begin{array}{c}\text { Processing Time tij of operation I on machine } \\
j=1\end{array}$ & $j=2$ \\
\hline 1 & 6 & 3 \\
2 & 2 & 9 \\
3 & 4 & 3 \\
4 & 1 & 8 \\
5 & 7 & 1 \\
6 & 4 & 5 \\
7 & 7 & 6 \\
\hline
\end{tabular}


In the "Shop Configuration" window, we select item "Flow Shop" among 'Single Machine', 'Parallel Machines' or 'Flow Shop' by double-clicking the item, as shown in Figure 3.

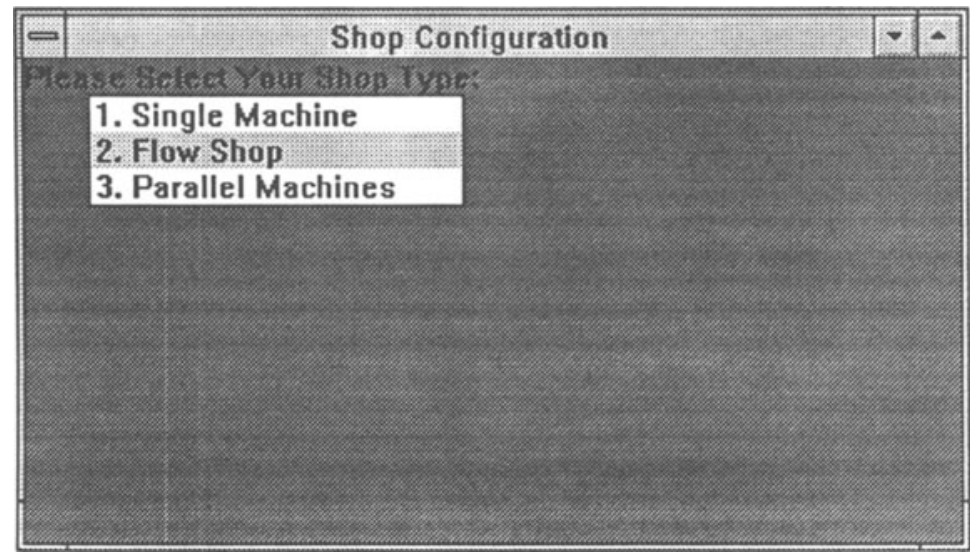

Figure 3 Shop type selection (Flow Shop)

Another window (Figure 4) will appear and ask the user the number of machines in his shop. Here the selection is " $m=2$ ".

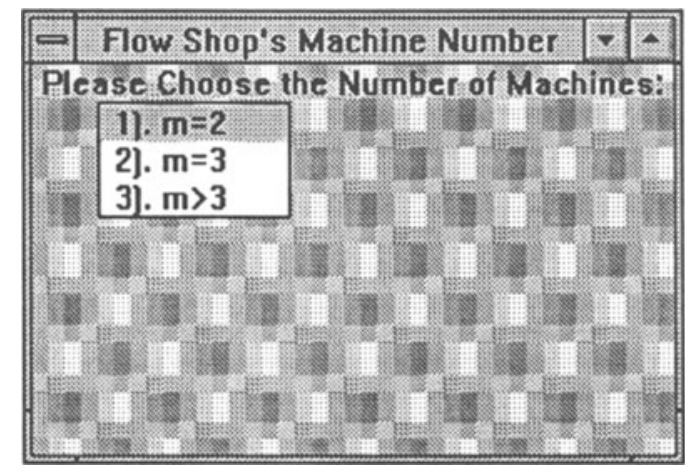

Figure 4 Window of selecting number of machines

Now comes another window (Figure 5). The function of this window is to let the user to enter the corresponding data of the shop. Operation number, processing time of operations on machine 1 and processing time of operations on machine 2 , respectively. 


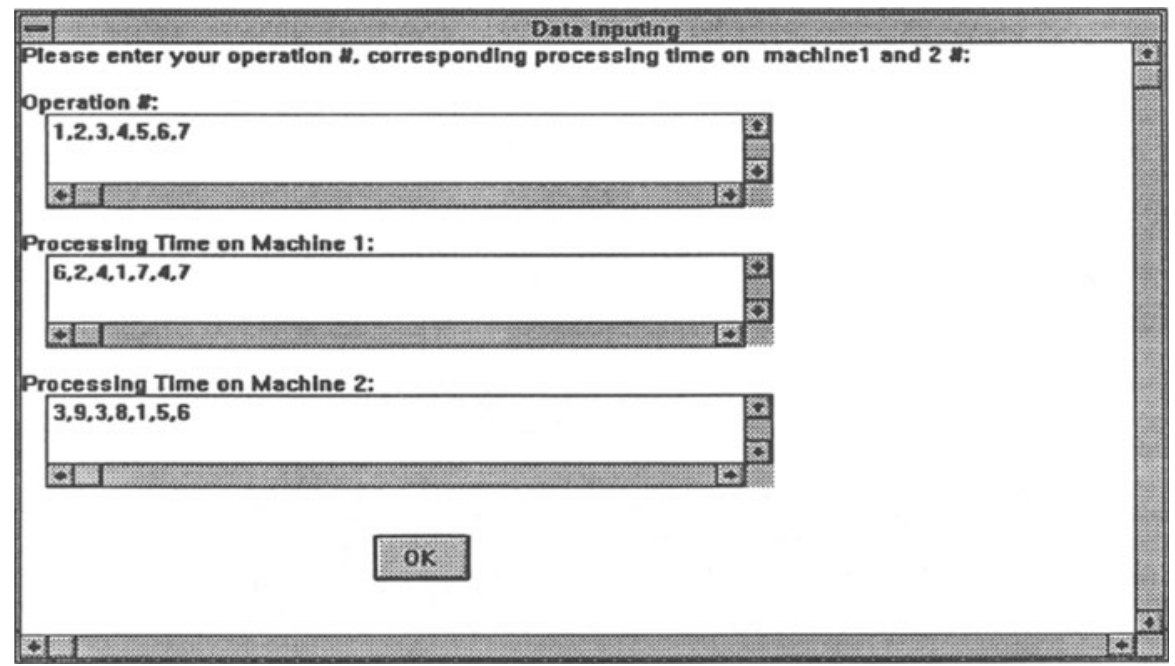

Figure 5 Window of data input of work shop

After the data have been input, the window (Figure 6) will show up to let the user to confirm the data, if the user want to change some data, just click on the button 'Back', the window (Figure 5) of data input will show up again. If the user has nothing to change, just click on the button 'Confirm'.

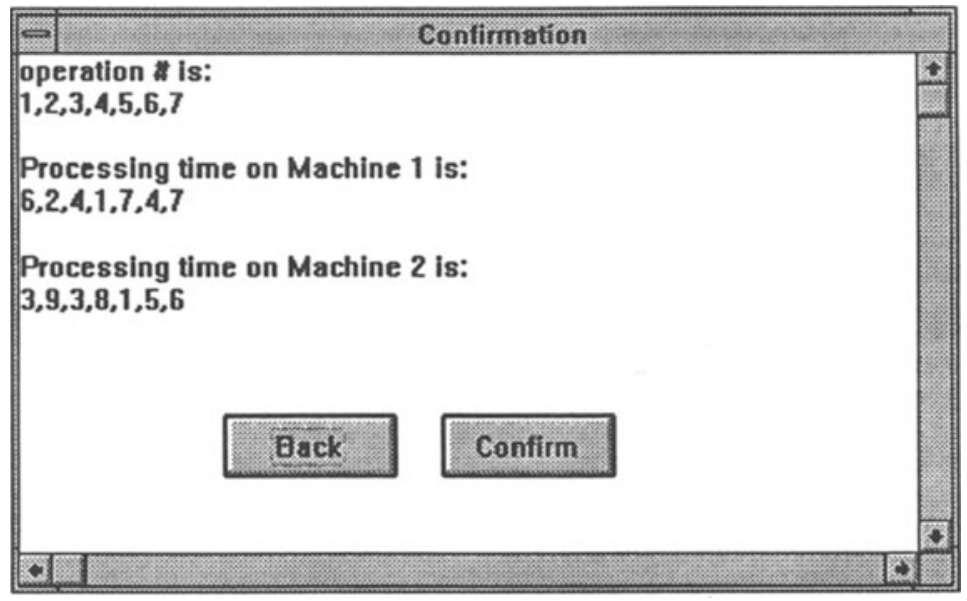

Figure 6 Confirmation of data input

After confirmation, the scheduling system will run and process the data according to Johnson's algorithm. During this time, a prompt window (Figure 7) will pop up. First the data will be written into a file in Microsoft Excel for first-step processing. 
Since Microsoft Excel has some functions on data sorting according to ascending or descending order of the data or according to some conditions, this function will save us lots of time in programming in C, so we make use of it and preprocess the data. Afterwards, the rearranged data is read by the meta-system, KnowledgePro. After reading, the meta-system will write and save the data into a text file and communicate the $\mathrm{C}$ program which will do the rest further processing of the data, at this time another window (Figure 8) will pop up to prompt user to wait for a few seconds.

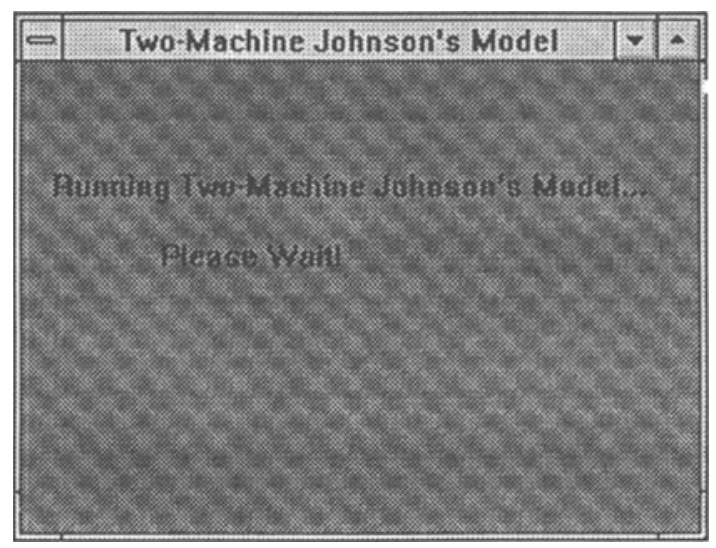

Figure 7 The prompt window

Now the system is running corresponding $\mathrm{C}$ program to calculate the optimal operation sequence according to the Johnson's algorithm. The result will come out with a window (Figure 8). 


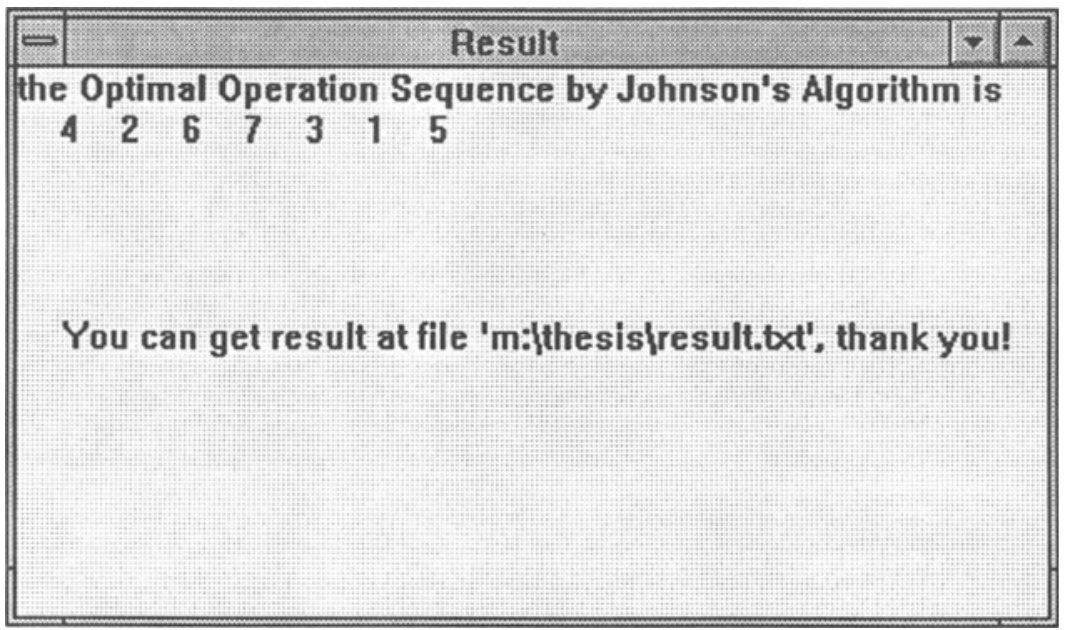

Figure 8 Window with the final result of Johnson's algorithm

\section{CONCLUSION}

A prototype system for automatic scheduling, which mainly includes the Metasystem, the expert system for scheduling selection, and subsystems of rules and algorithms is established. At present, the prototype system is limited to the static and deterministic problems, but it is very easy to be extended to dynamic and probabilistic scheduling problems, because the structure of the system is based on the concept of IIS. The problem of scheduling two-machine flow shop, which should be solved by rule of Johnson's, has been used to demonstrate how the system functions. What the user need to do is just to specify, for example, the user's shop type, the user's objectives and shop's parameters, the intelligence-inside scheduling system will choose appropriate rules or algorithms to run the corresponding calculations and tell user the final results. The intelligent development environment, KnowledgePro, has been selected for building of the system. It shows that KnowledgePro is a high level language, which provides object oriented programming, expert systems and multimedia facilities.

Alexander, S. M. (1978), An Expert system for the selection of scheduling Rules in a job shop, Computers and Industrial Engineering, Vol. 12, No. 3, pp. 167-171. 
Baker, K. R. (1974), Introduction to Sequencing and scheduling, wiley, New York. Bellman, R. E., Esogbue, A. O. (1982), and Nabeshima, I., Mathematical Aspect of Scheduling and Applications, Pergamon Press, Oxford.

Balckstone, J. H. Jr., Phillips D. T. and Hogg, G. L. (1982), A state-of-the-art survey of Dispatching Rules for Manufacturing Job Shop Operations, International Journal of Production Research, Vol. 20, No. 1, pp. 27-45.

Conway, R. W., Maxwell W. L., Miller L. W. (1967), Theory of Scheduling, Addison-Wesley.

Dorn J. and Froeschl K. A. (1993), Scheduling of Production Processes, Ellis Horwood Limited.

French, S. (1982), Sequencing and Scheduling: An Introduction to the mathematics of the job-shop, Wiley, New York,.

Gere, W. S. Jr. (1966), Heuristics in Job Shop Scheduling, Management Science, Vol. 13, No. 3, pp. 167-190.

Gursel A., Suer and Juan F. M. (1995), A Prototype Knowledge-Based System for Production Scheduling Problems, University of Puerto Rico., Proceedings of the first world congress on Intelligent manufacturing processes and systems, pp. 501-511.

KnowledgePro Windows(1991), Reference Manual, version 2.0, Knowledge Garden, October.

KnowledgePro Windows(1991), User Manual, version 2.0, Knowledge Garden, October.

Kusiak A. and Ahn J. (1992), Intelligent Scheduling of Automated Machining Systems, Intelligent Design and Manufacturing, (Ed. Kusiak A.) John Wiley \& Sons, Inc., pp. 421-447.

Kusiak A. and Wang J. (1994), A knowledge-based system for building scheduling models, Artificial Intelligence in optimal Design and Manufacturing (Ed. Dong Z.), P T R Prentice Hall Inc. pp. 173-202.

Panwallkar, S. S. and Iskander, W. (1977), A survey of Scheduling Rules, Operation Research, Vol. 25, No. 1, pp 45-61.

Pinedo M. (1995), Scheduling/theory, algorithms, and systems, Prentice Hall.

Schultz, C. R. (1998), An expediting Heuristic for the Shortest Processing Time Dispatching Rule, International Journal of Production Research, Vol. 27, No. 1 pp. 41-51.

Wang, K. (1991), Artificial Intelligence Applied to Mechanical Engineering, NTH.

Wang, K. (1995), SINTEF REPORT on Knowledge Integrated System in Manufacturing Engineering, Guest Lecture at Beijing Institute of PetrolChemical Technology.

Zweben M. and Fox M. S. (1994), Intelligent Scheduling, Morgan Kaufmann Publishers. 
Kesheng Wang, the professor of Department of Production and Quality Engineering, Norwegian University of Science and Technology (NTNU), He has received the B.S. (1968) and M.S.(1981) degree from The Shanghai University of Technology, Shanghai, China. and the Dr. Ing. (1988) from The Norwegian Institute of Technology (NTH), Trondheim, Norway. He has published two text books and over 60 technical papers in international journals and international conferences. He served as program committee member and session chairman in several international conferences, the chairman of Nordic and Baltic Summer School, and invited to given seminars at many universities and research institutes internationally. His research interests span structure design, kinematics and dynamics of robot manipulators, computer graphics simulation, manufacturing systems theory, mathematical modeling of mechanical systems, intelligent robotics, and expert systems and soft computing (ANN, FZ, Genetic Algorithms etc.) in production and engineering. Dr. Wang is the member of IFIP (International Federation of Information Process), TC5, WG. 5.3, the member of NAIA (Norwegian Artificial Intelligence Association) and The member of New York Academy of Science.

Wengfen Lin is a graduate student at the Department of Production and Quality Engineering, Norwegian University of Science and Technology (NTNU). He has received the B.S. (1992) from Tianjing University, China and M.S. (1997) from NTNU, Norway. 